\title{
Late Onset Post Transplant Lymphoproliferative Disorder (PTLd) - A Cause for Prolonged Fever In A Patient With Biliary Anastomotic Stricture- Need To Think Beyond Cholangitis!!!
}

\author{
Ashok Choudhury*, Apurva Pande, Binit Sureka and Chaggan Bihari Sharma \\ Department of Hepatology and Transplant Medicine, Institute of Liver and Biliary Sciences, India
}

Submission: May 27, 2017; Published: June 14, 2017

*Corresponding author: Ashok Choudhury, DM Hepatology, Assistant Professor; Department of Hepatology and Transplant Medicine, Institute of Liver and Biliary Sciences, D-1,Vasant Kunj, New Delhi 110070, India, Email: doctorashokchoudhury@gmail.com

\begin{abstract}
Post transplantation lymphoproliferative disorder (PTLD) is one of the less reported complications following liver transplantation (LT). With increased survival and longer follow-up of OLT recipients, the incidence of PTLD and especially, the number of EBV negative PTLD relative to the total number of PTLD, are increasing. Presentations can vary from a mild fever, fatigue to graft dysfunction. Reduced immunosuppression is the preferred therapy in majority; however additional rituximab, adoptive immunotherapy and chemotherapy needed in selective cases. The present case is a HCV related cirrhosis, on ERCP session for biliary stricture and presented after 20 months post live donor liver transplantation with continued fever for 3 weeks and on work up diagnosed to have PTLD in absence of EBV infection.
\end{abstract}

Keywords: Post transplant lymphoproliferative disorder; Ebstein Barr Virus; Orthotopic Liver Transplantation (OLT)

Abbreviations: PTLD: Post Transplant Lymphoproliferative Disorder; EBV: Ebstein Barr Virus; OLT: Orthotopic Liver Transplantation; CABG: Coronary Artery Bypass Graft; MMF: Mycophenolate Mofetil; LDLT: Liver Donor Liver Transplantation

\section{Introduction}

Post transplantation lymphoproliferative disorder (PTLD) is one of the less reported complications following liver transplantation (LT). With increased survival and longer followup of OLT recipients, the incidence of PTLD and especially, the number of EBV negative PTLD relative to the total number of PTLD, are increasing. Presentations can vary from a mild fever, fatigue to graft dysfunction. The incidence of PTLD was highest during the first 18 months post-OLT with cumulative incidences $0.5 \%, 0.9 \%$ and $1.1 \%$ at 6,12 and 18 months, respectively and in $80 \%$ of the cases is associated with Ebstein Barr Virus (EBV) infection. Duration and type of immunosuppression, CMV and HCV infection further increases the risk for PTLD. Reduced immunosuppression is the preferred therapy in majority, however additional rituximab, adoptive immunotherapy and chemotherapy needed in selective cases. The present case is a HCV related cirrhosis, on ERCP session for biliary stricture and presented after 20 months post live donor liver transplantation with continued fever for 3 weeks and on work up diagnosed to have PTLD in absence of EBV infection.
Case

A 63 years old male, diabetic, obese, post coronary artery bypass graft (CABG) surgery underwent live donor liver transplant (LDLT) in April 2014 for decompensated HCV related cirrhosis. Immediate postoperative period was uneventful and he was on triple immunosuppressant- steroid, tacroliumus and mycophenolate mofetil (MMF) till 3 months. Following this he was on tacrolimus with target tacroliums level between 6-8 and MMF of $1 \mathrm{gm}$ twice daily. At 6 months post LT he was diagnosed to have HCV recurrence in the graft biopsy with biliary anastomotic stricture. He received sofosbuvir and ribavirin for 24 weeks and acchieved SVR after 12 months. Undergoing ERCP and stenting for biliary complication every 6 weeks. After 20 months i.e. in December 2015 he presented with intermittent fever up to $101 \mathrm{~F}$-which responded to antipyretics, fatigability, in absence of any chills or rigor or night sweat or any evening rise of temperature. There was no jaundice, pruritus, clay color stool, cough, expectoration, joint pain, burning micturition or altered bowel habit $[1,2]$. 
His initial lab data revealed hemoglobin $(\mathrm{Hb})$ of $12.7 \mathrm{gm} /$ $\mathrm{dl}$, total leucocyte count (TLC) of $12.8 \times 103 /$ ccwith $70 \%$ neutrophil and 22\% lymphocytes and platelet count (PLT) of 1.61lac/cc. electrolyte, blood urea, serum creatinine was normal. Liver function tests (LFTs) showed serum bilirubin (S.Bil)-2.7mg/dl with direct being $0.9 \mathrm{mg} / \mathrm{dl}$, AST- 26IU/ $\mathrm{ml}$, ALT-24 IU/ml, serum alkaline phosphatase- $103 \mathrm{IU} / \mathrm{ml}$ and /gamaglutamyltranspeptidase of $175 \mathrm{IU} / \mathrm{ml}$, albumin/ globulin(Alb/Glob)-2.9/2.4. Blood and urine cultures were sterile (Figures 1-3).

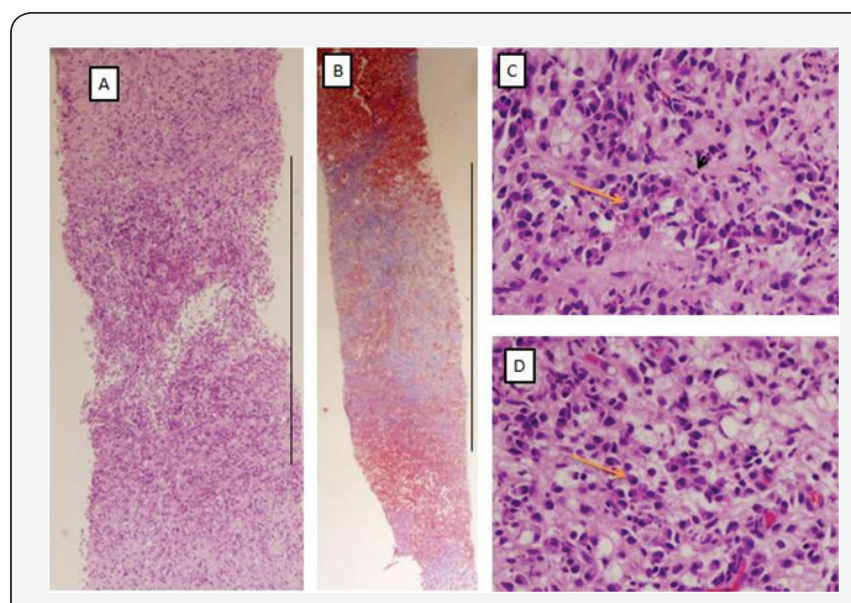

Figure 1: $(A, B)$ MR axial FIESTA images showing nodular hyperintense lesions in graft liver along the cut edge (arrows), coursing of segment $\mathrm{V}$ duct through the lesion with associated periportal lymphadenopathy (dashed arrows).

$(C, D)$ Diffusion-weighted MR images with b-value of 1000 showing bright signal in corresponding regions suggestive of diffusion restriction (arrowheads).

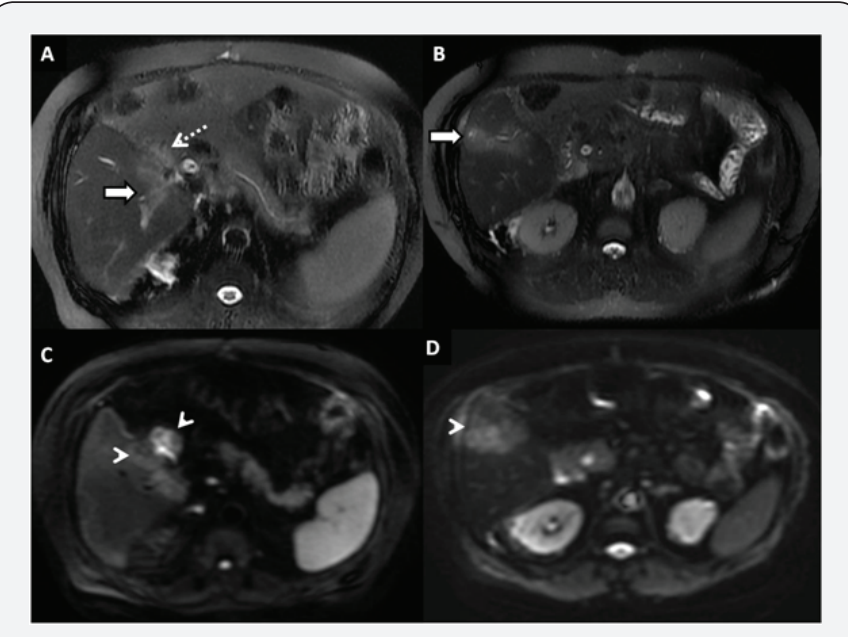

Figure 2: Liver biopsy: A. HE stained showing focal large area of inflammatory infiltrate, $40 \mathrm{X}$ B. MT stained section of the same biopsy showing background fibrosis in that lesion, 200X and $D$ \&E. Inflammatory infiltrate in different comprising of dense sheets of plasma cells (orange arrow), lymphoid cells and occasional neutrophils and black arrow head showing underlying background fibrosis, HE, 200X. E. Plasma cells are kappa positive $\mathrm{F}$. lambda positivity in plasma cells- Overall findings are consistent with Polymorphic polyclonal PTLD.

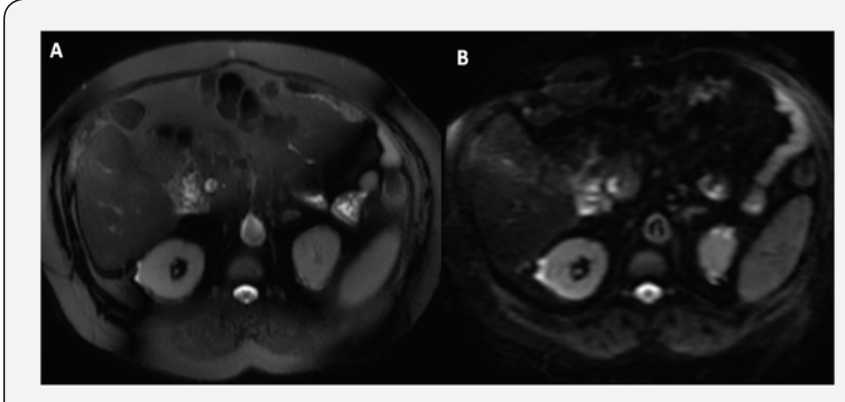

Figure 3: Figure: $(A, B)$ follow up MRI images.

On suspicion of fever due to biliary complication, a MRI with MRCP was done. It revealed the presence of ill-defined focal and confluent areas of T1 hypointensity /T2-hyperintensity along the portal tracts in graft liver predominantly involving segment-V showing minimal diffusion restriction with biliary radicals coursing through them along with a few diffusion restriction in periportal and peri-pancreatic lymph nodes. Hence raising the suspicion of PTLD. Contrast CT of thorax was non-contributory for the cause of fever. Peripheral smear showed no atypical cells, Bone marrow -normal with LDH-597 IU/ml. Targeted biopsy from the liver showed the normal liver acinar architecture with focal large area of mixed inflammatory infiltrate (A) comprising of dense sheets of plasma cells (orange arrow), lymphoid cells and occasional neutrophils and neovascularization in the background of dense collagenous stroma (B). Portal tract show mild mixed inflammation with periportal spillover with focal lymphocytic duct damage. IHC for EBV was negative but the plasma cells are kappa positive (E) and lambda positive (F), consistent with Polymorphic polyclonal PTLD. EBV DNA was undetectable and the tissue DNA extraction for EBV was also negative. The CMV DNA and HCV RNA were negative. It was again confirmed the EBV IgG was reactive both for donor and recipient in pre LT work up and presently also [3,4].

But being on low dose of immunosuppression, without any history of prior ACR requiring pulsing or increased dose of immunosuppression and post LT 20 months it is difficult to get a conclusion. Tacrolimus and MMF was stopped maintained on prednisolone $20 \mathrm{mg}$ and supportive medication. After 2 months on being serial follow up, he became afebrile, LFT normal and repeat MRI reveal-no lesion suggestive of resolution of PTLD.

\section{Discussion}

PTLD is recognized as one of the most devastating complications of following liver transplantation. PTLD following OLT in adults have reported frequencies in the $2 \%$ to $5.4 \%$ range. The Epstein-Barr virus (EBV) genome is found in the majority (>90\%) of B cell PTLD occurring early (within the first year) after transplantation. But more recent reports suggest an increasing frequency of EBV negative PTLD also.Various risk factors include first year post-transplant, EBV-seronegativity in the recipient and aggressive immunosuppression using anti-T cell antibodies 
such as OKT3 and ATG and possibly hepatitis C virus, alcoholic cirrhosis and older recipients. EBV capable of persisting in latent from after clearance of primary exposure, stimulating B-cell proliferation and transformation and persists in infected B-lymphocytes even after the clearance of the primary infection. Immunosuppression given after transplantation leads to a functional impairment of EBV- specific cytotoxic T lymphocytes leading to EBV-driven lymphoproliferation leading to PTLD [5].

The exact pathogenesis of EBV negative PTLD is not yet clear. Various postulations are like - undiscovered viruses, the loss of EBV infection or the initial EBV stimulated lymphoproliferation and subsequent new mutations over time, including those that result in EBV independent cell replication leading to PTLD. The EBV negative PTLD is considered to be a different entity and the number or EBV negative PTLD are likely to increase in the future with increased follow up and improved post LT survival. They tend to occur late after transplantation often present with nonspecific symptoms like weight loss, sweating and fever. Usually an extra-nodal disease, often in the allograft rarely widely disseminated monomorphic in histology with an aggressive clinical behavior and associated with the worse prognosis. One recent study suggested that that both the groups had about $40 \%$ mortality in the first year subsequent to diagnosis and had about a $50 \%$ survival at 5 years and did not differ much [6].

\section{Diagnosis}

A high index of suspicion based on clinical features in needed to make a diagnosis of PTLD. Serology i.e. IgM and IgG against early antigen (EA) and Epstein-Barr nuclear antigen are unreliable particularly on immunosuprresive therapy and more so in presence of preexisting antibodies. Viral load determination i.e. EBV DNA is the optimal way to perform, interpret and utilize quantitative EBV viral load assays for surveillance, diagnostic and disease monitoring purposes. Although EBV load is highly sensitive for predicting PTLD, the specificity in liver transplant recipients is only around $50 \%$. Detection of EBV nucleic acids or protein in tissue: by RNA in situ hybridization or immunohistochemistry using commercial antibodies against EBNA- 1, EBNA-2 and LMP-1 can detect EBV in fixed tissues in very low viral load or the EBV latent or lytic antigens.

CT of the chest and abdomen help to identify the concomitant other site lesion. Magnetic Resonance Imaging (MRI) may be a more suitable modality than CT both in radiation exposure as well as in precise delineation of lesion. Positron emission tomography-computerized tomography (PET-CT) is emerging to be a useful for diagnosis and guiding response to therapybut additional data are needed on its utility. Histopathologyremains the gold standard for PTLD diagnosis. Although excisional biopsy is preferred, needle biopsy is acceptable particularly for transplant cases. Immunophenotyping of T Cell or B Cell is essential to determine lineage and guide therapy particularly CD20. Clonality and EBV genome not routinely required [7].

\section{Treatment}

The treatment of PTLD remains a challenge. Currently, there is no unifying consensus but mostly personalized and decided case-to-case basis as controlled interventional studies are lacking. The general approach to therapy involves a step- wise strategy that starts with reduced immunosuppression, with plans for further escalation of treatment based largely on the clinical response and the histopathologic characteristics of the PTLD. Most patients would be expected to show evidence of a clinical response to reduced immunosuppression by $50 \%$ within 2-6 weeks.

The response ranges from 0 to $73 \%$ and complete response in $37 \%$, relapse in $17 \%$ that needs to be balanced to $32 \%$ risk acute rejection. Although neither EBV-seronegativity nor B cell histologic subtype influenced outcome, bulky disease, advanced stage and older age predicted lack of response. Complete or partial surgical resection with or without local radiotherapy in addition to reduced immunosuppression has been used in relapse cases leading to a long-termremission. Rituximab monotherapy gaining acceptance with overall response rate of $55 \%$ and improved progression free and overall survival. But no data on therapy for rituximab monotherapy relapse [8]. Presence one or more of the following components (age $>60$, Eastern Cooperative Oncology Group (ECOG) prognostic index of 2-4 and raised $\mathrm{LDH}$ ) from the prognostic model by Choquet et al. [6] predict poor survival after rituximab monotherapy and favors combination with chemotherapy as initial therapy.

The commonly used one is 4 weeks of rituximab therapy followed by four sequential cycles of rituximab/CHOP every 3 weeks (cyclophosphamide, doxorubicin, oncovin and prednisone). Another approach known as risk-stratified sequential therapy (RSST) is tailored approach, whereby patients who achieved complete remission with an initial four doses of rituximab received a second course of rituximab without chemotherapy. Acyclovir and ganciclovir with later being more effective have been used in the management of early PTLD, alone or in combination with immune globulin, but efficacy of this approach is uncertain and there is no evidence to support the use of antiviral agents in the absence of other interventions such as decreasing immunosuppression or anti-CD20 therapy. Adoptive immunotherapy using donor derived cloned EBVspecific cytotoxic T cells, Alpha interferon and Anti-IL6 therapy are other modalities that not routinely used.

Our patients is $>60$ years, diabetic, obese, CAD with post $\mathrm{CABG}$, prior HCV, large tumor with polymorphic feature on histology, more than $>1$ year post transplant and EBV negative is a unique case with great challenge. However improved with immunosuppression minimization another strong points for the case is that a patient of biliary complication presenting with prolonged fever despite adequate biliary drainage need a consideration of other possibility beyond cholangitis. In conclusion, EBV negative PTLD though uncommon but must 
be kept in mind while evaluation a post transplant patient who presents with prolonged favor and other non-specific symptoms.

\section{References}

1. Kremers WK, Devarbhavi HC, Wiesner RH (2006) Post-Transplant Lympho proliferative Disorders Following Liver Transplantation: Incidence, Risk Factors and Survival. Am J Transplant 6: 1017-1024.

2. Miyazono A, Okamoto Y, Nagasako H (2016) Multifocal Epstein-Barr Virus-Negative Post transplantation Lympho proliferative Disorder Treated With Reduction.

3. Kamdarb KY, Rooney CM and Heslop HE (2011) Post-transplant lymphoproliferative disease following liver transplantation. CurrOpin Organ Transplant 16(3): 274-280.

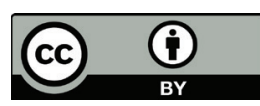

This work is licensed under Creative Commons Attribution 4.0 Licens DOI: 10.19080/OAJS.2017.04.555643
4. Mendizabal M, Marciano S (2013) Post-transplant lymphoproliferative disorder in adult liver transplant recipients: a South American multicenter experience. Clin Transplant 27(4): E469-477.

5. Patel H, Vogl DT, Aqui N (2007) Post transplant lymphoproliferative disorder in adult liver transplant recipients: a report of seventeen cases. Leuk Lymphoma 48(5): 885-891.

6. McLaughlin K, Wajstaub S, Marotta $P$ (2000) Increased risk for post transplant lympho prolife rative disease in recipients of liver transplants with hepatitis C. Liver Transpl 6: 570-574.

7. Gottschalk S, Rooney CM, Heslop HE (2005) Post-transplant lymphoproliferative disorders. Annu RevMed 56: 29-44.

8. Gan YJ, Razzouk BI, Su T, Sixbey JW (2002) A defective, rearranged Epstein-Barr virus genome in EBER-negative and EBER-positive Hodgkin's disease. Am J Pathol 160: 781-786.

\section{Your next submission with Juniper Publishers will reach you the below assets}

- Quality Editorial service

- Swift Peer Review

- Reprints availability

- E-prints Service

- Manuscript Podcast for convenient understanding

- Global attainment for your research

- Manuscript accessibility in different formats

( Pdf, E-pub, Full Text, Audio)

- Unceasing customer service

Track the below URL for one-step submission https://juniperpublishers.com/online-submission.php 